\title{
Robust Stabilization for Uncertain Takagi-Sugeno Fuzzy Continuous Model with Time-Delay Based on Razumikhin Theorem
}

\author{
Yassine Manai and Mohamed Benrejeb \\ Additional information is available at the end of the chapter
}

http://dx.doi.org/10.5772/48075

\section{Introduction}

Fuzzy control systems have experienced a big growth of industrial applications in the recent decades, because of their reliability and effectiveness. Many researches are investigated on the Takagi-Sugeno models [1], [2] and [3] last decades. Two classes of Lyapunov functions are used to analysis these systems: quadratic Lyapunov functions and non-quadratic Lyapunov ones which are less conservative than first class. Many researches are investigated with non-quadratic Lyapunov functions [4]-[6], [7].

Recently, Takagi-Sugeno fuzzy model approach has been used to examine nonlinear systems with time-delay, and different methodologies have been proposed for analysis and synthesis of this type of systems [1]-[11], [12]-[13]. Time delay often occurs in many dynamical systems such as biological systems, chemical system, metallurgical processing system and network system. Their existences are frequently a cause of infeasibility and poor performances.

The stability approaches are divided into two classes in term of delay. The fist one tries to develop delay independent stability criteria. The second class depends on the delay size of the time delay, and it called delay dependent stability criteria. Generally, delay dependent class gives less conservative stability criteria than independent ones.

Two classes of Lyapunov-Razumikhin function are used to analysis these systems: quadratic Lyapunov-Razumikhin function and non-quadratic Lyapunov- Razumikhin ones. The use of first class brings much conservativeness in the stability test. In order to reduce the conservatism entailed in the previous results using quadratic function.

As the information about the time derivatives of membership function is considered by the PDC fuzzy controller, it allows the introduction of slack matrices to facilitate the stability 
analysis. The relationship between the membership function of the fuzzy model and the fuzzy controllers is used to introduce some slack matrix variables. The boundary information of the membership functions is brought to the stability condition and thus offers some relaxed stability conditions [5].

In this chapter, a new stability conditions for time-delay Takagi-Sugeno fuzzy systems by using fuzzy Lyapunov-Razumikhin function are presented. In addition, a new stabilization conditions for Takagi Sugeno time-delay uncertain fuzzy models based on the use of fuzzy Lyapunov function are presented. This criterion is expressed in terms of Linear Matrix Inequalities (LMIs) which can be efficiently solved by using various convex optimization algorithms [8],[9]. The presented methods are less conservative than existing results.

The organization of the chapter is as follows. In section 2, we present the system description and problem formulation and we give some preliminaries which are needed to derive results. Section 3 will be concerned to stability and stabilization analysis for T-S fuzzy systems with Parallel Distributed Controller (PDC). An observer approach design is derived to estimate state variables. Section 5 will be concerned to stabilization analysis for timedelay T-S fuzzy systems based on Razumikhin theorem. Next, a new robust stabilization condition for uncertain system with time delay is given in section 6. Illustrative examples are given in section 7 for a comparison of previous results to demonstrate the advantage of proposed method. Finally section 8 makes conclusion.

Notation: Throughout this chapter, a real symmetric matrix $S>0$ denotes $S$ being a positive definite matrix. The superscript " $\mathrm{T}$ " is used for the transpose of a matrix.

\section{System description and preliminaries}

Consider an uncertain T-S fuzzy continuous model with time-delay for a nonlinear system as follows:

$$
\begin{aligned}
& \text { IF } z_{1}(t) \text { is } M_{i 1} \text { and ... and } z_{p}(t) \text { is } M_{i p} \\
& \text { THEN }\left\{\begin{array}{l}
\dot{x}(t)=\left(A_{i}+\Delta A_{i}\right) x(t)+\left(D_{i}+\Delta D_{i}\right) x\left(t-\tau_{i}(t)\right)+\left(B_{i}+\Delta B_{i}\right) u(t) \\
x(t)=\phi(t), t \in[-\tau, 0]
\end{array}\right.
\end{aligned}
$$

where $M_{i j}(i=1,2, \ldots, r, j=1,2, \ldots, p)$ is the fuzzy set and $r$ is the number of model rules; $x(t) \in \mathfrak{R}^{n}$ is the state vector, $u(t) \in \mathfrak{R}^{m}$ is the input vector, $A_{i} \in \mathfrak{R}^{n \times n}, D_{i} \in \mathfrak{R}^{n \times n}, B_{i} \in \mathfrak{R}^{n \times m}$, and $z_{1}(t), \ldots, z_{p}(t)$ are known premise variables, $\phi(t)$ is a continuous vector-valued initial function on $[-\tau, 0]$; the time-delay $\tau(t)$ may be unknown but is assumed to be smooth function of time.. $\Delta A_{i}, \Delta D_{i}$ and $\Delta B_{i}$ are time-varying matrices representing parametric uncertainties in the plant model. These uncertainties are admissibly norm-bounded and structured.

$$
0 \leq \tau(t) \leq \tau, \quad \dot{\tau}(t) \leq d \prec 1,
$$

where $\tau \succ 0$ and $d$ are two scalars. 
The final outputs of the fuzzy systems are:

$$
\begin{gathered}
\dot{x}(t)=\sum_{i=1}^{r} h_{i}(z(t))\left\{\left(A_{i}+\Delta A_{i}\right) x(t)+\left(D_{i}+\Delta D_{i}\right) x\left(t-\tau_{i}(t)\right)+\left(B_{i}+\Delta B_{i}\right) u(t)\right\} \\
x(t)=\phi(t), \quad t \in[-\tau, 0]
\end{gathered}
$$

where

$$
\begin{gathered}
z(t)=\left[z_{1}(t) z_{2}(t) \ldots z_{p}(t)\right] \\
h_{i}(z(t))=w_{i}(z(t)) / \sum_{i=1}^{r} w_{i}(z(t)), w_{i}(z(t))=\prod_{j=1}^{p} M_{i j}\left(z_{j}(t)\right) \text { for all } \underline{t} .
\end{gathered}
$$

The term $M_{i 1}\left(z_{j}(t)\right)$ is the grade of membership of $z_{j}(t)$ in $M_{i 1}$

Since $\quad\left\{\begin{array}{l}\sum_{i=1}^{r} w_{i}(z(t)) \succ 0 \\ w_{i}(z(t)) \geq 0, \quad i=1,2, \ldots, r\end{array}\right.$

we have $\left\{\begin{array}{l}\sum_{i=1}^{r} h_{i}(z(t))=1 \\ h_{i}(z(t)) \geq 0, \quad i=1,2, \ldots, r\end{array} \quad\right.$ for all $t$.

The time derivative of premise membership functions is given by:

$$
\dot{h}_{i}(z(t))=\frac{\partial h_{i}}{\partial z(t)} \cdot \frac{\partial z(t)}{\partial x(t)} \cdot \frac{d x(t)}{d t}=\sum_{l=1}^{s} v_{i l} \xi_{i l} \times \frac{d x(t)}{d t}
$$

We have the following property:

$$
\sum_{k=1}^{r} \dot{h}_{k}(z(t))=0
$$

Consider a PDC fuzzy controller based on the derivative membership function and given by the equation (4)

$$
u(t)=-\sum_{i=1}^{r} h_{i}(z(t)) F_{i} x(t)-\sum_{m=1}^{r} \dot{h}_{m}(z(t)) K_{m} x(t)
$$

The fuzzy controller design consists to determine the local feedback gains $F_{i}$, and $K_{m}$ in the consequent parts. The state variables are determined by an observer which detailed in next section. 
By substituting (4) into (2), the closed-loop fuzzy system without time-delay can be represented as:

$$
\begin{gathered}
\dot{x}(t)=\sum_{i=1}^{r} \sum_{j=1}^{r} h_{i}(z(t)) h_{j}(z(t))\left\{\left[A_{\Delta i}-B_{\Delta i} F_{j}-\sum_{m=1}^{r} \dot{h}_{m}(z(t)) B_{\Delta i} K_{m}\right] x(t)+D_{\Delta i} x\left(t-\tau_{i}(t)\right)\right\} \\
x(t)=\phi(t), \quad t \in[-\tau, 0],
\end{gathered}
$$

where $A_{\Delta i}=A_{i}+\Delta A_{i} ; D_{\Delta i}=D_{i}+\Delta D_{i}$ and $B_{\Delta i}=B_{i}+\Delta B_{i}$

The system without uncertainties is given by equation

$$
\begin{gathered}
\dot{x}(t)=\sum_{i=1}^{r} \sum_{j=1}^{r} h_{i}(z(t)) h_{j}(z(t))\left\{\left[A_{i}-B_{i} F_{j}-\sum_{m=1}^{r} \dot{h}_{m}(z(t)) B_{i} K_{m}\right] x(t)+D_{i} x\left(t-\tau_{i}(t)\right)\right\} \\
x(t)=\phi(t), \quad t \in[-\tau, 0],
\end{gathered}
$$

The open-loop system is given by the equation (7),

$$
\begin{gathered}
\dot{x}(t)=\sum_{i=1}^{r} h_{i}(z(t))\left(A_{\Delta i} x(t)+D_{\Delta i} x\left(t-\tau_{i}(t)\right)\right) \\
x(t)=\phi(t), \quad t \in[-\tau, 0],
\end{gathered}
$$

\section{Assumption 1}

The time derivative of the premises membership function is upper bounded such that $\left|\dot{h}_{k}\right| \leq \phi_{k}$, for $k=1, \ldots, r$, where, $\phi_{k}, k=1, \ldots, r$ are given positive constants.

\section{Assumption 2}

The matrices denote the uncertainties in the system and take the form of

$$
\left\{\begin{array}{l}
\Delta A_{i}=D_{a_{i}} F_{a_{i}}(t) E_{a_{i}} \\
\Delta B_{i}=D_{b_{i}} F_{b_{i}}(t) E_{b_{i}}
\end{array}\right.
$$

where $D_{a_{i}}, D_{b_{i}}, E_{a_{i}}$ and $E_{b_{i}}$ are known constant matrices and $F_{a_{i}}(t)$ and $F_{b_{i}}(t)$ are unknown matrix functions satisfying :

$$
\left\{\begin{array}{l}
F_{a_{i}}^{T}(t) F_{a_{i}}(t) \leq I, \forall t \\
F_{b_{i}}^{T}(t) F_{b_{i}}(t) \leq I, \forall t
\end{array}\right.
$$

where $\mathrm{I}$ is an appropriately dimensioned identity matrix. 
Lemma 1 (Boyd et al. Schur complement [16])

Given constant matrices $\Omega_{1}, \Omega_{2}$ and $\Omega_{3}$ with appropriate dimensions, where $\Omega_{1}=\Omega_{1}^{T}$ and $\Omega_{2}=\Omega_{2}^{T}$, then

$$
\Omega_{1}+\Omega_{3}^{T} \Omega_{2}^{-1} \Omega_{3} \prec 0
$$

if and only if

$$
\left[\begin{array}{cc}
\Omega_{1} & \Omega_{3}^{T} \\
* & -\Omega_{2}
\end{array}\right] \prec 0 \text { or }\left[\begin{array}{cc}
-\Omega_{2} & \Omega_{3} \\
* & \Omega_{1}
\end{array}\right] \prec 0
$$

Lemma 2 (Peterson and Hollot [2])

Let $Q=Q^{T}, H, E$ and $F(t)$ satisfying $F^{T}(t) F(t) \leq I$ are appropriately dimensional matrices then the follow-ing inequality

$$
Q+H F(t) E+E^{T} F^{T}(t) H^{T} \prec 0
$$

is true, if and only if the following inequality holds for any $\lambda \succ 0$

$$
Q+\lambda^{-1} H H^{T}+\lambda E^{T} E \prec 0
$$

Theorem 1 (Razumikhin Theorem)[5]

Suppose $u, v, w: \mathfrak{R}^{+} \rightarrow \mathfrak{R}^{+}$are continuous, non-decreasing functions satisfying $u(s) \succ 0$, $v(s) \succ 0$ and $w(s) \succ 0$ for $s \succ 0, u(0)=v(0)=0$, and $v$ strictly increasing. If there exist a continuous function $V: \mathfrak{R} \times \Re^{n} \rightarrow \mathfrak{R}$ and a continuous non-decreasing function $p(s) \succ s$ for $s \succ 0$ such that

$$
\begin{gathered}
u(|x|) \leq V(t, x) \leq v(|x|), \quad \forall t \in \mathfrak{R}, x \in \mathfrak{R}^{n}, \\
\dot{V}(t, x) \leq-w(|x|) \quad \text { if } V(t+\sigma, x(t+\sigma)) \leq p(V(t, x)), \quad \forall \sigma \in[-\tau, 0],
\end{gathered}
$$

then the solution $x \equiv 0$ of $(7)$ is uniformly asymptotically stable.

\section{Lemma 3 [6]}

Assume that $a \in \mathfrak{R}^{n_{a}}, b \in \mathfrak{R}^{n_{b}}, N \in \mathfrak{R}^{n_{a} \times n_{b}}$ are defined on the interval $\Omega$. Then, for any matrices $X \in \mathfrak{R}^{n_{a} \times n_{a}}, Y \in \mathfrak{R}^{n_{a} \times n_{b}}$ and $Z \in \mathfrak{R}^{n_{b} \times n_{b}}$, the following holds:

$$
-2 \int_{\Omega} a^{T}(\alpha) N b(\alpha) d \alpha \leq \int_{\Omega}^{[}\left[\begin{array}{l}
a(\alpha) \\
b(\alpha)
\end{array}\right]^{T}\left[\begin{array}{cc}
X & Y-N \\
Y^{T}-N^{T} & Z
\end{array}\right]\left[\begin{array}{l}
a(\alpha) \\
b(\alpha)
\end{array}\right] d \alpha,
$$


where $\left[\begin{array}{cc}X & Y \\ Y^{T} & Z\end{array}\right] \geq 0$.

\section{Lemma 4 [9]}

The unforced fuzzy time delay system described by (7) with $\mathrm{u}=0$ is uniformly asymptotically stable if there exist matrices $P \succ 0, S_{i} \succ 0, X_{a i}, X_{d i}, Z_{a i j}, Z_{d i j}$, and $Y_{i}$, such that the following LMIs hold:

$$
\begin{gathered}
{\left[\begin{array}{cc}
P A_{i}+A_{i}^{T} P+\tau\left(X_{a i}+X_{d i}\right)+(2 \tau+1) P+Y_{i}+Y_{i}^{T} & -P D_{i} \\
Y_{i}^{T}-D_{i}^{T} P & -S_{i}
\end{array}\right] \prec 0} \\
S_{i} \leq P \\
A_{j}^{T} Z_{a i j} A_{j} \leq P \\
D_{j}^{T} Z_{d i j} D_{j} \leq P \\
{\left[\begin{array}{cc}
X_{a i} & Y_{i} \\
Y_{i}^{T} & Z_{a i j}
\end{array}\right] \geq 0} \\
{\left[\begin{array}{ll}
X_{d i} & Y_{i} \\
Y_{i}^{T} & Z_{d i j}
\end{array}\right] \geq 0}
\end{gathered}
$$

\section{Basic stability and stabilization conditions}

In order to design an observer for state variables, this section introduce two theorem developed for continuous TS fuzzy model for open-loop and closed-loop. First, consider the open-loop system without time-delay given by equation(17).

$$
\dot{x}(t)=\sum_{i=1}^{r} h_{i}(z(t)) A_{i} x(t)
$$

The main approach for T-S fuzzy model stability is given in theorem follows. This approach is based on introduction of $\varepsilon$ parameter which influences the stability region.

\section{Theorem 2 [17]}

Under assumption 1 and for $0 \leq \varepsilon \leq 1$, the Takagi Sugeno fuzzy system (17) is stable if there exist positive definite symmetric matrices $P_{k}, k=1,2, \ldots, r$, matrix $R=R^{T}$ such that the following LMIs hold. 


$$
\begin{gathered}
P_{k}+R \succ 0, \quad k \in\{1, \ldots, r\} \\
P_{j}+\mu R \succ 0, \quad j \in\{1, \ldots, r\} \\
P_{\phi}+\frac{1}{2}\left\{A_{i}^{T}\left(P_{j}+\mu R\right)+\left(P_{j}+\mu R\right) A_{i}\right. \\
\left.+A_{j}^{T}\left(P_{i}+\mu R\right)+\left(P_{i}+\mu R\right) A_{j}\right\} \prec 0, \quad i \leq j
\end{gathered}
$$

where $i, j=1,2, \ldots, r$ and $P_{\phi}=\sum_{k=1}^{r} \phi_{k}\left(P_{k}+R\right)$ and $\mu=1-\varepsilon$

\section{Proof}

The proof of this theorem is given in detailed in article published in [17].

The closed-loop system without time delay is given by equation (21)

$$
\dot{x}(t)=\sum_{i=1}^{r} h_{i}(z(t)) h_{i}(z(t)) G_{i i} x(t)+2 \sum_{i=1}^{r} \sum_{i \prec j} h_{i}(z(t)) h_{j}(z(t))\left\{\frac{G_{i j}+G_{j i}}{2}\right\} x(t),
$$

where

$$
G_{i j}=A_{i}-B_{i} F_{j} \text { and } G_{i i}=A_{i}-B_{i} F_{i}
$$

In this section we define a fuzzy Lyapunov function and then consider stability conditions. A sufficient stability condition, for ensuring stability is given follows.

\section{Theorem 2[18]}

Under assumption 1, and assumption 2 and for given $0 \leq \varepsilon \leq 1$, the Takagi-Sugeno system (21) is stable if there exist positive definite symmetric matrices $P_{k}, k=1,2, \ldots, r$, and $R$, matrices $F_{1}, \ldots, F_{r}$ such that the following LMIs hols.

$$
\begin{gathered}
P_{k}+R \succ 0, \quad k \in\{1, \ldots, r\} \\
P_{j}+\mu R \geq 0, \quad j=1,2, \ldots, r \\
P_{\phi}+\left\{G_{i i}^{T}\left(P_{k}+\mu R\right)+\left(P_{k}+\mu R\right) G_{i i}\right\} \prec 0, \\
i, k \in\{1, \ldots, r\} \\
\left\{\frac{G_{i j}+G_{j i}}{2}\right\}^{T}\left(P_{k}+\mu R\right)+\left(P_{k}+\mu R\right)\left\{\frac{G_{i j}+G_{j i}}{2}\right\} \prec 0, \\
\text { for } i, j, k=1,2, \ldots, r \text { such that } i \prec j
\end{gathered}
$$


where

$$
G_{i j}=A_{i}-B_{i} F_{j}, G_{i i}=A_{i}-B_{i} F_{i}
$$

And $P_{\phi}=\sum_{k=1}^{r} \phi_{k}\left(P_{k}+R\right)$

\section{Observer design for T-S fuzzy continuous model}

In order to determine state variables of system, this section gives a solution by the mean of fuzzy observer design.

A stabilizing observer-based controller can be formulated as follow:

$$
\begin{aligned}
& \hat{\dot{x}}(t)=\sum_{j=1}^{r} h_{i}(z(t))\left\{A_{i} \hat{x}(t)+B_{i} u(t)+L_{j}\left(C_{i} \hat{x}(t)-y(t)\right)\right\} \\
& u(t)=\sum_{j=1}^{r} h_{j}(z(t)) F_{j} \hat{x}(t)
\end{aligned}
$$

The closed-loop fuzzy system can be represented as:

$$
\begin{gathered}
\dot{x}(t)=\sum_{i=1}^{r} \sum_{j=1}^{r} h_{i}(z(t)) h_{j}(z(t))\left\{\left(A_{i}-B_{i} F_{j}\right)-\sum_{\rho=1}^{r} \dot{h}_{\rho}(z(t))\left(H_{\rho}+R\right)\right\} x(t) \\
+\sum_{i=1}^{r} \sum_{j=1}^{r} h_{i}(z(t)) h_{j}(z(t))\left\{B_{i} F_{j}+\sum_{\rho=1}^{r} \dot{h}_{\rho}(z(t))\left(H_{\rho}+R\right)\right\} e(t) \\
\dot{e}(t)=\sum_{i=1}^{r} \sum_{j=1}^{r} h_{i}(z(t)) h_{j}(z(t))\left\{A_{i}-K_{i} C_{j}\right\} e(t)
\end{gathered}
$$

The augmented system is represented as follows:

$$
\begin{aligned}
\dot{x}_{a}(t) & =\sum_{i=1}^{r} \sum_{j=1}^{r} h_{i}(z(t)) h_{j}(z(t)) G_{i j} x_{a}(t) \\
& =\sum_{j=1}^{r} h_{i}(z(t)) h_{j}(z(t)) G_{i i} x_{a}(t)+2 \sum_{i=1}^{r} \sum_{i \prec j}^{r} h_{i}(z(t)) h_{j}(z(t))\left\{\frac{G_{i j}+G_{j i}}{2}\right\} x_{a}(t)
\end{aligned}
$$

where

$$
\begin{aligned}
& x_{a}(t)=\left[\begin{array}{l}
x(t) \\
e(t)
\end{array}\right] \\
& G_{i j}=\left[\begin{array}{cc}
A_{i}-B_{i} F_{j}-\sum_{\rho=1}^{r} \dot{h}_{\rho} B_{i}\left(H_{\rho}+R\right) & B_{i} F_{j}+\sum_{\rho=1}^{r} \dot{h}_{\rho} B_{i}\left(H_{\rho}+R\right) \\
0 & A_{i}-K_{i} C_{j}
\end{array}\right]
\end{aligned}
$$


By applying Theorem 2[18] in the augmented system (29) we derive the following Theorem.

\section{Theorem 3}

Under assumption 1 and for given $0 \leq \mu \leq 1$, the Takagi-Sugeno system (29) is stable if there exist positive definite symmetric matrices $P_{k}, k=1,2, \ldots, r$, and $R$, matrices $F_{1}, \ldots, F_{r}$ such that the following LMIs hols.

$$
\begin{gathered}
P_{k}+R \succ 0, \quad k \in\{1, \ldots, r\} \\
P_{j}+\mu R \geq 0, \quad j=1,2, \ldots, r \\
P_{\phi}+\left\{G_{i i}^{T}\left(P_{k}+\mu R\right)+\left(P_{k}+\mu R\right) G_{i i}\right\} \prec 0, \\
i, k \in\{1, \ldots, r\} \\
\left\{\frac{G_{i j}+G_{j i}}{2}\right\}^{T}\left(P_{k}+\mu R\right)+\left(P_{k}+\mu R\right)\left\{\frac{G_{i j}+G_{j i}}{2}\right\} \prec 0, \\
\text { for } i, j, k=1,2, \ldots, r \text { such that } i \prec j
\end{gathered}
$$

where

$$
G_{i j}=\left[\begin{array}{cc}
A_{i}-B_{i} F_{j}-\sum_{\rho=1}^{r} \dot{h}_{\rho} B_{i}\left(H_{\rho}+R\right) & B_{i} F_{j}+\sum_{\rho=1}^{r} \dot{h}_{\rho} B_{i}\left(H_{\rho}+R\right) \\
0 & A_{i}-K_{i} C_{j}
\end{array}\right]
$$

And $P_{\phi}=\sum_{k=1}^{r} \phi_{k}\left(P_{k}+R\right)$

\section{Proof}

The result follows immediately from the Theorem 2[18].

\section{Stabilization of continuous T-S Fuzzy model with time-delay}

The aim of this section is to prove the asymptotic stability of the time-delay system (6) based on the combination between Lyapunov theory and the Razumikhin theorem [5].

\section{Theorem 4}

Under assumption 1 and for given $0 \leq \varepsilon \leq 1$, the unforced fuzzy time delay system described by (7) with $u=0$ is uniformly asymptotically stable if there exist matrices $P_{k} \succ 0, k=1,2, \ldots, r$, $S_{i} \succ 0, X_{a i j}, X_{d i}, Z_{a i j}, Z_{d i j}, Y_{i}$, and $X$, such that the following LMIs hold: 


$$
\left[\begin{array}{cc}
P_{\beta}+\left(P_{k}+\varepsilon X\right) G_{i j}+G_{i j}^{T}\left(P_{k}+\varepsilon X\right) & \\
+\tau\left(X_{a i j}+X_{d i}\right)+(2 \tau+1)\left(P_{k}+\varepsilon X\right)+Y_{i}+Y_{i}^{T} & -\left(P_{k}+\varepsilon X\right) D_{i} \\
Y_{i}^{T}-D_{i}^{T}\left(P_{k}+\varepsilon X\right) & -S_{i}
\end{array}\right] \prec 0
$$

where $P_{\beta}=\sum_{k=1}^{r} \beta_{k}\left(P_{k}+\varepsilon X\right)$.

$$
G_{i j}=A_{i}-B_{i} F_{j}
$$

$$
\begin{gathered}
S_{i} \leq\left(P_{k}+\varepsilon X\right) \\
G_{i j}^{T} Z_{a i j} G_{i j} \leq\left(P_{k}+\varepsilon X\right) \\
D_{j}^{T} Z_{d i j} D_{j} \leq\left(P_{k}+\varepsilon X\right) \\
{\left[\begin{array}{cc}
X_{a i j} & Y_{i} \\
Y_{i}^{T} & Z_{a i j}
\end{array}\right] \geq 0} \\
{\left[\begin{array}{cc}
X_{d i} & Y_{i} \\
Y_{i}^{T} & Z_{d i j}
\end{array}\right] \geq 0}
\end{gathered}
$$

\section{Proof}

Let consider the fuzzy Lyapunov function as

$$
\begin{gathered}
V(x)=x^{T}(t) V_{k}(x) x(t) \\
V_{k}(x)=\sum_{k=1}^{r} h_{k}\left(P_{k}+\varepsilon X\right)
\end{gathered}
$$

Given the matrix property, clearly,

$$
\lambda_{\min }\left(P_{k}+\varepsilon X\right)\|x(t)\|^{2} \leq x^{T}(t)\left(P_{k}+\varepsilon X\right) x(t) \leq \lambda_{\max }\left(P_{k}+\varepsilon X\right)\|x(t)\|^{2},
$$

where $\lambda_{\min (\max )}$ denotes the smallest (largest) eigenvalue of the matrix.

Finding the maximum value of $\sum_{k=0}^{r} h_{k} x^{T}(t)\left(P_{k}+\varepsilon X\right) x(t)$ is equivalent to determining the maximum value of $\sum_{k=0}^{r} h_{k} \lambda_{\max }\left(P_{k}+\varepsilon X\right)$. 
Finding the minimum value of $\sum_{k=0}^{r} h_{k} x^{T}(t)\left(P_{k}+\varepsilon X\right) x(t)$ is equivalent to determining the minimum value of $\sum_{k=0}^{r} h_{k} \lambda_{\min }\left(P_{k}+\varepsilon X\right)$.

Define

$$
\begin{aligned}
& \kappa_{1}=\min _{k} \sum_{k=0}^{r} h_{k} \lambda_{\text {max }}\left(P_{k}+\varepsilon X\right) \text { for } 0 \leq k \leq r, \\
& \kappa_{2}=\max _{k} \sum_{k=0}^{r} h_{k} \lambda_{\min }\left(P_{k}+\varepsilon X\right) \text { for } 0 \leq k \leq r .
\end{aligned}
$$

Then,

$$
\kappa_{1}\|x(t)\|^{2} \leq \sum_{k=1}^{r} x^{T}(t)\left(P_{k}+\varepsilon X\right) x(t) \leq \kappa_{2}\|x(t)\|^{2}
$$

In the following, we will prove the asymptotic stability of the time-delay system (7) based on the Razumikhin theorem [5].

Since

$$
x(t)-x\left(t-\tau_{i}(t)\right)=\int_{t-\tau_{i}(t)}^{t} \dot{x}(s) d s,
$$

The state equation of (7) with $u=0$ can be rewritten as

$$
\dot{x}(t)=\sum_{i=1}^{r} \sum_{j=1}^{r} h_{i} h_{j}\left[\left(G_{i j}+D_{i}\right) x(t)-D_{i} \int_{t-\tau_{i}(t)}^{t} \dot{x}(s) d s\right]
$$

where $G_{i j}=A_{i}-B_{i} F_{j}$

The derivative of $V$ along the solutions of the unforced system (7) with $u=0$ is thus given by

$$
\begin{gathered}
\dot{V}=x^{T}(t) \sum_{k=1}^{r} \dot{h}_{k}\left(P_{k}+\varepsilon X\right) x(t)+2 x^{T}(t) \sum_{i=1}^{r} h_{i}\left(P_{i}+\varepsilon X\right) \dot{x}(t)=\Upsilon_{1}(x, t)+\Upsilon_{2}(x, t) \\
\Upsilon_{1}(x, t)=x^{T}(t) \sum_{k=1}^{r} \dot{h}_{k}\left(P_{k}+\varepsilon X\right) x(t) \\
\Upsilon_{2}(x, t)=2 x^{T}(t) \sum_{k=1}^{r} h_{k}\left(P_{k}+\varepsilon X\right) \dot{x}(t)=2 x^{T}(t) \sum_{k=1}^{r} h_{k}\left(P_{k}+\varepsilon X\right) \times \sum_{i=1}^{r} \sum_{j=1}^{r} h_{i} h_{j}\left[\left(G_{i j}+D_{i}\right) x(t)-D_{i} \int_{t-\tau_{i}(t)}^{t} \dot{x}(s) d s\right] .
\end{gathered}
$$

Then, based on assumption 1 , an upper bound of $\Upsilon_{1}(x, z)$ obtained as:

$$
\Upsilon_{1}(x, z) \leq \sum_{k=1}^{r} \beta_{k} \cdot x(t)^{T}\left(P_{k}+\varepsilon X\right) x(t)
$$


and for $\Upsilon_{2}(x, t)$ we can written as,

$$
\begin{aligned}
\Upsilon_{2}(x, t)= & 2 \sum_{i=1}^{r} \sum_{j=1}^{r} h_{i} h_{j} x^{T} \sum_{k=1}^{r} h_{k}\left(P_{k}+\varepsilon X\right)\left(G_{i j}+D_{i}\right) x(t)-\sum_{i=1}^{r} \sum_{j=1}^{r} h_{i} h_{j} \int_{t-\tau_{i}(t)}^{t}\left\{2 x^{T}(t) \sum_{k=1}^{r} h_{k}\left(P_{k}+\varepsilon X\right) D_{i}\right. \\
& \left.\times \sum_{v=1}^{r} \sum_{\zeta=1}^{r} h_{v}(s) h_{\varsigma}(s)\left[G_{v \varsigma} x(s)+D_{\varsigma} x\left(s-\tau_{j}(s)\right)\right] d s\right\} \\
\Upsilon_{2}(x, t)= & 2 \sum_{i=1}^{r} \sum_{j=1}^{r} \sum_{k=1}^{r} h_{i} h_{j} h_{k} x^{T}\left\{\left(P_{k}+\varepsilon X\right)\left(G_{i j}+D_{i}\right)\right\} x(t)-\sum_{i=1}^{r} \sum_{j=1}^{r} \sum_{k=1}^{r} h_{i} h_{j} h_{k} \int_{t-\tau_{i}(t)}^{t}\left\{2 x^{T}(t)\left(P_{k}+\varepsilon X\right) D_{i}\right. \\
& \left.\times \sum_{v=1}^{r} \sum_{\zeta=1}^{r} h_{v}(s) h_{\varsigma}(s)\left[G_{v \varsigma} x(s)+D_{\varsigma} x\left(s-\tau_{j}(s)\right)\right] d s\right\}
\end{aligned}
$$

Using the bounding method in(10), by setting $a=x(t)$ and $b=G_{i j} x(s)$, we have

$$
\begin{aligned}
& -\int_{t-\tau_{i}(t)}^{t} 2 x^{T}(t)\left(P_{k}+\varepsilon X\right) D_{i} \times \sum_{v=1}^{r} \sum_{\varsigma=1}^{r} h_{v}(s) h_{\varsigma}(s) G_{v \varsigma} x(s) d s \\
& \leq \tau_{i}(t) x^{T}(t) X_{a i} x(t)+2 x^{T}(t)\left(Y_{i}-\left(P_{k}+\varepsilon X\right) D_{i}\right) \times \int_{t-\tau_{i}(t)}^{t} \sum_{v=1}^{r} \sum_{\varsigma=1}^{r} h_{v}(s) h_{\varsigma}(s) G_{v \varsigma} x(s) d s \\
& +\int_{t-\tau_{i}(t)}^{t} \sum_{v=1}^{r} \sum_{\zeta=1}^{r} h_{v}(s) h_{\varsigma}(s) x^{T}(s) G_{v \varsigma}^{T} Z_{a i v} G_{v \varsigma} x(s) d s
\end{aligned}
$$

For any matrices $X_{a v}, Y_{v}$ and $Z_{a i v}$ satisfying

$$
\left[\begin{array}{cc}
X_{a v} & Y_{v} \\
Y_{v}^{T} & Z_{a i v}
\end{array}\right] \geq 0
$$

Similarly, it holds that

$$
\begin{aligned}
& -\int_{t-\tau_{i}(t)}^{t} 2 x^{T}(t)\left(P_{k}+\varepsilon X\right) D_{i} \sum_{j=1}^{r} h_{j}(s) D_{j} x\left(s-\tau_{j}(s)\right) d s \\
& \leq \tau_{i}(t) x^{T}(t) X_{d i} x(t)+2 x^{T}(t)\left(Y_{i}-\left(P_{k}+\varepsilon X\right) D_{i}\right) \int_{t-\tau_{i}(t)}^{t} \sum_{j=1}^{r} h_{j}(s) D_{j} x\left(s-\tau_{j}(s)\right) d s \\
& +\int_{t-\tau_{i}(t)}^{t} \sum_{j=1}^{r} h_{j}(s) x^{T}\left(s-\tau_{j}(s)\right) D_{j}^{T} Z_{d i j} D_{j} x\left(s-\tau_{j}(s)\right) d s
\end{aligned}
$$

For any matrices $X_{d i}, Y_{i}$ and $Z_{d i j}$ satisfying 


$$
\left[\begin{array}{cc}
X_{d i} & Y_{i} \\
Y_{i}^{T} & Z_{d i j}
\end{array}\right] \geq 0
$$

Hence, substituting (44) and (45) into (43), we have

$$
\begin{aligned}
\dot{V} \leq & P_{\beta}+\sum_{i=1}^{r} \sum_{j=1}^{r} \sum_{k=1}^{r} h_{i} h_{j} h_{k} x^{T}(t)\left[2\left(P_{k}+\varepsilon X\right)\left(G_{i j}+D_{i}\right)+\tau\left(X_{a i}+X_{d i}\right)\right] x(t) \\
& +\sum_{i=1}^{r} \sum_{k=1}^{r} h_{i} h_{k} 2 x^{T}(t)\left(Y_{i}-\left(P_{k}+\varepsilon X\right) D_{i}\right) \times \int_{t-\tau_{i}(t)}^{t} \sum_{v=1}^{r} \sum_{\varsigma=1}^{r} h_{v}(s) h_{\varsigma}(s)\left[G_{v \varsigma} x(s)+D_{v} x\left(s-\tau_{v}(s)\right)\right] d s \\
& +\sum_{i=1}^{r} \sum_{k=1}^{r} h_{i} h_{k} \int_{t-\tau_{i}(t)}^{t} \sum_{v=1}^{r} \sum_{\zeta=1}^{r} h_{v}(s) h_{\varsigma}(s) x^{T}(s) G_{v \varsigma}^{T} Z_{a i v} G_{v \varsigma} x(s) d s \\
& +\sum_{i=1}^{r} \sum_{k=1}^{r} h_{i} h_{k} \int_{t-\tau_{i}(t)}^{t} \sum_{j=1}^{r} h_{j}(s) x^{T}\left(s-\tau_{j}(s)\right) D_{j}^{T} Z_{d i j} D_{j} x\left(s-\tau_{j}(s)\right) d s \\
\leq P_{\beta} & +\sum_{i=1}^{r} \sum_{j=1}^{r} h_{i} h_{j} x^{T}(t)\left[\left(P_{k}+\varepsilon X\right) G_{i j}+G_{i j}^{T}\left(P_{k}+\varepsilon X\right)+Y_{i}+Y_{i}^{T}+\tau\left(X_{a i j}+X_{d i}\right)\right] x(t) \\
& +\sum_{i=1}^{r} h_{i}\left[x^{T}(t)\left(Y_{i}-\left(P_{k}+\varepsilon X\right) D_{i}\right) S_{i}^{-1}\left(Y_{i}-\left(P_{k}+\varepsilon X\right) D_{i}\right)^{T} x(t)+x^{T}\left(t-\tau_{i}(t)\right) S_{i} x\left(t-\tau_{i}(t)\right)\right] \\
& +\sum_{i=1}^{r} h_{i} \times \int_{t-\tau_{i}(t)}^{t} \sum_{v=1}^{r} \sum_{\zeta=1}^{r} h_{v}(s) h_{\varsigma}(s) x^{T}(s) G_{v \varsigma}^{T} Z_{a i v} G_{v \varsigma} x(s) d s \\
& +\sum_{i=1}^{r} h_{i} \int_{t-\tau_{i}(t)}^{t} \sum_{j=1}^{r} h_{j}(s) x^{T}\left(s-\tau_{j}(s)\right) D_{j}^{T} Z_{d i j} D_{j} x\left(s-\tau_{j}(s)\right) d s
\end{aligned}
$$

Note that, by Shur complement, the LMI in (34) implies $L_{i}(\delta) \prec 0$ for a sufficiently small scalar $\delta \succ 0$, where

$$
\begin{aligned}
& L_{i}(\delta)=P_{\beta}+\left(P_{k}+\varepsilon X\right) G_{i j}+G_{i j}^{T}\left(P_{k}+\varepsilon X\right)+Y_{i}+Y_{i}^{T}+\tau\left(X_{a i j}+X_{d i}\right) \\
& +\left(Y_{i}-\left(P_{k}+\varepsilon X\right) D_{i}\right) S_{i}^{-1}\left(Y_{i}-\left(P_{k}+\varepsilon X\right) D_{i}\right)^{T} x(t)+(2 \tau+1+\tau \delta)(1+\delta)\left(P_{k}+\varepsilon X\right)
\end{aligned}
$$

In order to use the Razumikhin Theorem, suppose $V(x(t+\sigma)) \prec(1+\delta) V(x(t))$ for $\sigma \in[-\tau, 0]$. Then, if the LMIs in (35)-(39) also hold, we have from (46) that

$$
\begin{aligned}
\dot{V} \leq & \sum_{i=1}^{r} \sum_{j=1}^{r} h_{i} h_{j} x^{T}(t)\left[\left(P_{k}+\varepsilon X\right) G_{i j}+G_{i j}^{T}\left(P_{k}+\varepsilon X\right)+Y_{i}+Y_{i}^{T}+\tau\left(X_{a i j}+X_{d i}\right)\right] x(t) \\
& +\sum_{i=1}^{r} h_{i}\left[x^{T}(t)\left(Y_{i}-\left(P_{k}+\varepsilon X\right) D_{i}\right) S_{i}^{-1}\left(Y_{i}-\left(P_{k}+\varepsilon X\right) D_{i}\right)^{T} x(t)+x^{T}(t)(1+\delta)\left(P_{k}+\varepsilon X\right) x(t)\right] \\
& +\sum_{i=1}^{r} h_{i} \tau_{i}(t) x^{T}(t)(1+\delta)\left(P_{k}+\varepsilon X\right) x(t)+\sum_{i=1}^{r} h_{i} \int_{t-\tau_{i}(t)}^{t} x^{T}(s)(1+\delta)\left(P_{k}+\varepsilon X\right) x(s) d s
\end{aligned}
$$




$$
\begin{gathered}
\leq \sum_{i=1}^{r} h_{i} x^{T}(t)\left[\left(P_{k}+\varepsilon X\right) A_{i}+A_{i}^{T}\left(P_{k}+\varepsilon X\right)+Y_{i}+Y_{i}^{T}+\tau\left(X_{a i}+X_{d i}\right)\right] x(t) \\
+\sum_{i=1}^{r} h_{i}\left[x^{T}(t)\left(Y_{i}-\left(P_{k}+\varepsilon X\right) D_{i}\right) S_{i}^{-1}\left(Y_{i}-\left(P_{k}+\varepsilon X\right) D_{i}\right)^{T} x(t)+x^{T}(t)(1+\delta)\left(P_{k}+\varepsilon X\right) x(t)\right] \\
+\tau x^{T}(t)(1+\delta)\left(P_{k}+\varepsilon X\right) x(t)+\tau x^{T}(t)(1+\delta)^{2}\left(P_{k}+\varepsilon X\right) x(t) \\
=\sum_{i=1}^{r} h_{i} x^{T}(t) L_{i}(\delta) x(t) \\
\prec 0
\end{gathered}
$$

which shows the motion of the unforced system (7) with $u=0$ is uniformly asymptotically stable. This completes the proof.

\section{Robust stability condition with PDC controller}

Consider the closed-loop system (5). A sufficient robust stability condition for Time-delay system is given follow.

\section{Theorem 5}

Under assumption 1, and assumption 2 and for given $0 \leq \varepsilon \leq 1$, the Takagi-Sugeno system (5) is stable if there exist positive definite symmetric matrices $P_{k}, k=1,2, \ldots, r$, and $R$, matrices $F_{1}, \ldots, F_{r}$ such that the following LMIs hols.

$$
\begin{gathered}
P_{k}+R \succ 0, \quad k \in\{1, \ldots, r\} \\
P_{j}+\mu R \geq 0, \quad j=1,2, \ldots, r \\
{\left[\begin{array}{cccc}
\Phi_{1} & \left(P_{k}+\mu R\right) D_{a i} & \left(P_{k}+\mu R\right) D_{b i} & \left(P_{k}+\mu R\right)\left(D_{d i} \Delta_{d i} E_{d i}\right) \\
* & -\lambda I & 0 & 0 \\
* & * & -\lambda I & 0 \\
* & * & * & 0
\end{array}\right] \prec 0} \\
i, k \in\{1, \ldots, r\}
\end{gathered}
$$

with

$$
\Phi_{1}=P_{\phi}+\bar{G}_{i i}^{T}\left(P_{k}+\mu R\right)+\left(P_{k}+\mu R\right) \bar{G}_{i i}+\lambda\left(P_{k}+\mu R\right)\left[E_{a i}^{T} E_{a i}+\left(E_{b i} F_{i}\right)^{T} E_{b i} F_{i}\right]
$$




$$
\begin{aligned}
& {\left[\begin{array}{ccc}
\Phi_{2} & & \\
* & -\lambda I & 0 \\
* & * & -\lambda I
\end{array}\right] \prec 0} \\
& {\left[\begin{array}{cccc}
\Phi_{2} & \left(P_{k}+\mu R\right)\left(D_{a i}+D_{a j}\right) & \left(P_{k}+\mu R\right)\left(D_{b i}+D_{b j}\right) & \left(P_{k}+\mu R\right)\left(D_{d i} \Delta_{d i} E_{d i}\right) \\
* & -\lambda I & 0 & 0 \\
* & * & -\lambda I & 0 \\
* & * & * & 0
\end{array}\right] \prec 0} \\
& \begin{array}{l}
i, k \in\{1, \ldots, r\} \\
\text { for } i, j, k=1,2, \ldots, r \text { such that } i \prec j
\end{array}
\end{aligned}
$$

with

$$
\begin{aligned}
\Phi_{2} & =\left(\frac{\bar{G}_{i j}+\bar{G}_{j i}}{2}\right)^{T}\left(P_{k}+\mu R\right)+\left(P_{k}+\mu R\right)\left(\frac{\bar{G}_{i j}+\bar{G}_{j i}}{2}\right)+\lambda\left(P_{k}+\mu R\right)\left[\left(E_{a i}+E_{a j}\right)^{T}\left(E_{a i}+E_{a j}\right)\right. \\
& \left.+\left(E_{b i} F_{j}+E_{b j} F_{i}\right)^{T}\left(E_{b i} F_{j}+E_{b j} F_{i}\right)^{T}\right]
\end{aligned}
$$

where $\bar{G}_{i j}=\left[A_{i}-B_{i} F_{j}-\sum_{m=1}^{r} \dot{h}_{m}(z(t)) B_{i} K_{m}\right], G_{i i}=\left[A_{i}-B_{i} F_{i}-\sum_{m=1}^{r} \dot{h}_{m}(z(t)) B_{i} K_{m}\right], \mu=1-\varepsilon$, and $P_{\phi}=\sum_{k=1}^{r} \phi_{k}\left(P_{k}+R\right)$

\section{Proof}

Let consider the Lyapunov function in the following form:

$$
V(x(t))=\sum_{k=1}^{r} h_{k}(z(t)) \cdot V_{k}(x(t))
$$

with

$$
V_{k}(x(t))=x^{T}(t)\left(P_{k}+\mu R\right) x(t), \quad k=1,2, \ldots, r
$$

where

$$
P_{k}=P_{k}^{T}, R=R^{T}, 0 \leq \varepsilon \leq 1, \mu=1-\varepsilon, \text { and }\left(P_{k}+\mu R\right) \geq 0, \quad k=1,2, \ldots, r .
$$

The time derivative of $V(x(t))$ with respect to $t$ along the trajectory of the system (21) is given by:

$$
\dot{V}(x(t))=\sum_{k=1}^{r} \dot{h}_{k}(z(t)) V_{k}(x(t))+\sum_{k=1}^{r} h_{k}(z(t)) \dot{V}_{k}(x(t))
$$


The equation (52) can be rewritten as,

$$
\begin{aligned}
\dot{V}(x(t)) & =x^{T}(t)\left(\sum_{k=1}^{r} \dot{h}_{k}(z(t))\left(P_{k}+\mu R\right)\right) x(t)+\dot{x}^{T}(t)\left(\sum_{k=1}^{r} h_{k}(z(t))\left(P_{k}+\mu R\right)\right) x(t) \\
& +x^{T}(t)\left(\sum_{k=1}^{r} h_{k}(z(t))\left(P_{k}+\mu R\right)\right) \dot{x}(t)
\end{aligned}
$$

By substituting (5) into (53), we obtain,

$$
\dot{V}(x(t))=\Upsilon_{1}(x, z)+\Upsilon_{2}(x, z)+\Upsilon_{3}(x, z)
$$

where

$$
\begin{aligned}
& \Upsilon_{1}(x, z)=x^{T}(t)\left(\sum_{k=1}^{r} \dot{h}_{k}(z(t)) \cdot\left(P_{k}+\mu R\right)\right) x(t) \\
& \Upsilon_{2}(x, z)=x^{T}(t) \sum_{k=1}^{r} \sum_{i=1}^{r} h_{k}(z(t)) h_{i}^{2}(z(t)) \times\left\{\bar{G}_{i i}^{T}\left(P_{k}+\mu R\right)+\left(P_{k}+\mu R\right) \bar{G}_{i i}\right\} x(t) \\
& +x^{T}(t) \sum_{k=1}^{r} \sum_{i=1}^{r} h_{k}(z(t)) h_{i}^{2}(z(t)) \times\left\{\left(\left[\begin{array}{ll}
D_{a i} & D_{b i}
\end{array}\right]\left[\begin{array}{cc}
\Delta_{a i} & 0 \\
0 & \Delta_{b i}
\end{array}\right]\left[\begin{array}{l}
E_{a i} \\
-E_{b i} F_{i}
\end{array}\right]\right)^{T}\left(P_{k}+\mu R\right)\right. \\
& \left.+\left(P_{k}+\mu R\right)\left(\left[\begin{array}{ll}
D_{a i} & D_{b i}
\end{array}\right]\left[\begin{array}{cc}
\Delta_{a i} & 0 \\
0 & \Delta_{b i}
\end{array}\right]\left[\begin{array}{l}
E_{a i} \\
-E_{b i} F_{i}
\end{array}\right]\right)\right\} x(t) \\
& +x^{T}\left(t-\tau_{i}(t)\right) \sum_{k=1}^{r} \sum_{i=1}^{r} h_{i}(z(t)) h_{k}(z(t))\left\{\left(D_{d i} \Delta_{d i} E_{d i}\right)^{T}\left(P_{k}+\mu R\right)\right\} x(t) \\
& +x^{T}(t) \sum_{k=1}^{r} \sum_{i=1}^{r} h_{i}(z(t)) h_{k}(z(t))\left(P_{k}+\mu R\right)\left(D_{d i} \Delta_{d i} E_{d i}\right) x\left(t-\tau_{i}(t)\right) \\
& \Upsilon_{2}(x, z)=\sum_{k=1}^{r} \sum_{i=1}^{r} h_{k}(z(t)) h_{i}^{2}(z(t)) \times \eta^{T} \Sigma_{i i} \eta
\end{aligned}
$$

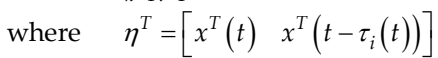

$$
\begin{aligned}
& \Sigma_{i i}=\left[\begin{array}{cc}
\Pi_{1} & \left(P_{k}+\mu R\right)\left(D_{d i} \Delta_{d i} E_{d i}\right) \\
\left\{\left(D_{d i} \Delta_{d i} E_{d i}\right)^{T}\left(P_{k}+\mu R\right)\right\} & 0
\end{array}\right] \\
& \text { with } \quad \Pi_{1}=\left\{\bar{G}_{i i}^{T}\left(P_{k}+\mu R\right)+\left(P_{k}+\mu R\right) \bar{G}_{i i}\right\} \\
& +\left\{\left(\left[\begin{array}{ll}
D_{a i} & D_{b i}
\end{array}\right]\left[\begin{array}{cc}
\Delta_{a i} & 0 \\
0 & \Delta_{b i}
\end{array}\right]\left[\begin{array}{l}
E_{a i} \\
-E_{b i} F_{i}
\end{array}\right]\right)^{T}\left(P_{k}+\mu R\right)+\left(P_{k}+\mu R\right)\left(\left[\begin{array}{ll}
D_{a i} & D_{b i}
\end{array}\right]\left[\begin{array}{cc}
\Delta_{a i} & 0 \\
0 & \Delta_{b i}
\end{array}\right]\left[\begin{array}{l}
E_{a i} \\
-E_{b i} F_{i}
\end{array}\right]\right)\right\} x(t) \\
& \text { where } \bar{G}_{i i}=\left[A_{i}-B_{i} F_{i}-\sum_{m=1}^{r} \dot{h}_{m}(z(t)) B_{i} K_{m}\right]
\end{aligned}
$$




$$
\begin{aligned}
& \Upsilon_{3}(x, z)=x(t)^{T} \sum_{k=1}^{r} \sum_{i=1}^{r} \sum_{i \prec j} h_{k}(z(t)) h_{i}(z(t)) h_{j}(z(t)) \times\left\{\left(\frac{\bar{G}_{i j}+\bar{G}_{j i}}{2}\right)^{T}\left(P_{k}+\mu R\right)+\left(P_{k}+\mu R\right)\left(\frac{\left.\bar{G}_{i j}+\bar{G}_{j i}\right)}{2}\right)\right\} x(t) \\
& +x(t)^{T} \sum_{k=1}^{r} \sum_{i=1}^{r} \sum_{i \prec j} h_{k}(z(t)) h_{i}(z(t)) h_{j}(z(t)) \times\left\{\left(\left[\begin{array}{ll}
D_{a i} & D_{b i}
\end{array}\right]\left[\begin{array}{cc}
\Delta_{a i} & 0 \\
0 & \Delta_{b i}
\end{array}\right]\left[\begin{array}{l}
E_{a i} \\
-E_{b i} F_{j}
\end{array}\right]\right)^{T}\left(P_{k}+\mu R\right)\right. \\
& \left.+\left(P_{k}+\mu R\right)\left(\left[\begin{array}{ll}
D_{a i} & D_{b i}
\end{array}\right]\left[\begin{array}{cc}
\Delta_{a i} & 0 \\
0 & \Delta_{b i}
\end{array}\right]\left[\begin{array}{l}
E_{a i} \\
-E_{b i} F_{j}
\end{array}\right]\right)\right\} x(t)+x(t)^{T} \sum_{k=1}^{r} \sum_{i=1}^{r} \sum_{i \prec j} h_{k}(z(t)) h_{i}(z(t)) h_{j}(z(t)) \\
& \times\left\{\left(\left[\begin{array}{ll}
D_{a j} & D_{b j}
\end{array}\right]\left[\begin{array}{cc}
\Delta_{a j} & 0 \\
0 & \Delta_{b j}
\end{array}\right]\left[\begin{array}{l}
E_{a j} \\
-E_{b j} F_{i}
\end{array}\right]\right)^{T}\left(P_{k}+\mu R\right)+\left(P_{k}+\mu R\right)\left(\left[\begin{array}{ll}
D_{a j} & D_{b j}
\end{array}\right]\left[\begin{array}{cc}
\Delta_{a j} & 0 \\
0 & \Delta_{b j}
\end{array}\right]\left[\begin{array}{l}
E_{a j} \\
-E_{b j} F_{i}
\end{array}\right]\right)\right\} x(t) \\
& +x^{T}\left(t-\tau_{i}(t)\right) \sum_{k=1}^{r} \sum_{i=1}^{r} h_{i}(z(t)) h_{k}(z(t))\left\{\left(D_{d i} \Delta_{d i} E_{d i}\right)^{T}\left(P_{k}+\mu R\right)\right\} x(t) \\
& +x^{T}(t) \sum_{k=1}^{r} \sum_{i=1}^{r} h_{i}(z(t)) h_{k}(z(t))\left(P_{k}+\mu R\right)\left(D_{d i} \Delta_{d i} E_{d i}\right) x\left(t-\tau_{i}(t)\right) \\
& \text { where } \bar{G}_{i j}=\left[A_{i}-B_{i} F_{j}-\sum_{m=1}^{r} \dot{h}_{m}(z(t)) B_{i} K_{m}\right] \\
& \Upsilon_{3}(x, z)=\sum_{k=1}^{r} \sum_{i=1}^{r} \sum_{i<j} h_{k}(z(t)) h_{i}(z(t)) h_{j}(z(t)) \times \eta^{T} \Sigma_{i j} \eta \\
& \text { where } \eta^{T}=\left[\begin{array}{ll}
x^{T}(t) & x^{T}\left(t-\tau_{i}(t)\right)
\end{array}\right] \\
& \Sigma_{i j}=\left[\begin{array}{cc}
\Pi_{2} & \left(P_{k}+\mu R\right)\left(D_{d i} \Delta_{d i} E_{d i}\right) \\
\left\{\left(D_{d i} \Delta_{d i} E_{d i}\right)^{T}\left(P_{k}+\mu R\right)\right\} & 0
\end{array}\right] \\
& \text { with } \left.\Pi_{2}=\left\{\left(\frac{\bar{G}_{i j}+\bar{G}_{j i}}{2}\right)^{T}\left(P_{k}+\mu R\right)+\left(P_{k}+\mu R\right)\left(\frac{\bar{G}_{i j}+\bar{G}_{j i}}{2}\right)\right\}\right] \\
& +\left\{\left(\left[\begin{array}{ll}
D_{a i} & D_{b i}
\end{array}\right]\left[\begin{array}{cc}
\Delta_{a i} & 0 \\
0 & \Delta_{b i}
\end{array}\right]\left[\begin{array}{l}
E_{a i} \\
-E_{b i} F_{j}
\end{array}\right]\right)^{T}\left(P_{k}+\mu R\right)+\left(P_{k}+\mu R\right)\left(\left[\begin{array}{ll}
D_{a i} & D_{b i}
\end{array}\right]\left[\begin{array}{cc}
\Delta_{a i} & 0 \\
0 & \Delta_{b i}
\end{array}\right]\left[\begin{array}{l}
E_{a i} \\
-E_{b i} F_{j}
\end{array}\right]\right)\right\} \\
& +\left\{\left(\left[\begin{array}{ll}
D_{a j} & D_{b j}
\end{array}\right]\left[\begin{array}{cc}
\Delta_{a j} & 0 \\
0 & \Delta_{b j}
\end{array}\right]\left[\begin{array}{l}
E_{a j} \\
-E_{b j} F_{i}
\end{array}\right]\right)^{T}\left(P_{k}+\mu R\right)+\left(P_{k}+\mu R\right)\left(\left[\begin{array}{ll}
D_{a j} & D_{b j}
\end{array}\right]\left[\begin{array}{cc}
\Delta_{a j} & 0 \\
0 & \Delta_{b j}
\end{array}\right]\left[\begin{array}{l}
E_{a j} \\
-E_{b j} F_{i}
\end{array}\right]\right)\right\}
\end{aligned}
$$

Then, based on assumption 1, an upper bound of $\Upsilon_{1}(x, z)$ obtained as:

$$
\Upsilon_{1}(x, z) \leq \sum_{k=1}^{r} \phi_{k} \cdot x(t)^{T}\left(P_{k}+\mu R\right) x(t)
$$
Based on (3), it follows that $\sum_{k=1}^{r} \dot{h}_{k}(z(t)) \varepsilon R=\bar{R}=0$ where $\mathrm{R}$ is any symmetric matrix of
proper dimension.

Adding $\bar{R}$ to (55), then 


$$
\Upsilon_{1}(x, z) \leq \sum_{k=1}^{r} \phi_{k} \cdot x(t)^{T}\left(P_{k}+R\right) x(t)
$$

Then,

$$
\dot{V}(x(t)) \leq \sum_{k=1}^{r} \phi_{k} x^{T}(t)\left(P_{k}+R\right) x(t)+\Upsilon_{2}(x, z)+\Upsilon_{3}(x, z)
$$

If

$$
\begin{aligned}
& {\left[\begin{array}{cc}
H_{11} & \left(P_{k}+\mu R\right) D_{d i} \Delta_{d i} E_{d i} \\
E_{d i}^{T} \Delta_{d i}^{T} D_{d i}^{T}\left(P_{k}+\mu R\right) & 0
\end{array}\right] \prec 0} \\
& \text { where } \quad H_{11}=\sum_{k=1}^{r} \phi_{k}\left(P_{k}+R\right)+\bar{G}_{i i}^{T}\left(P_{k}+\mu R\right)+\left(P_{k}+\mu R\right) \bar{G}_{i i} \\
& +\left\{\left(\left[\begin{array}{ll}
E_{a i} \\
-E_{b i} F_{i}
\end{array}\right]\right)^{T}\left(\left[\begin{array}{ll}
D_{a i} & D_{b i}
\end{array}\right]\right)^{T}\left(P_{k}+\mu R\right)+\left(P_{k}+\mu R\right)\left[\begin{array}{ll}
D_{a i} & D_{b i}
\end{array}\right]\left[\begin{array}{cc}
\Delta_{a i} & 0 \\
0 & \Delta_{b i}
\end{array}\right]\left[\begin{array}{l}
E_{a i} \\
-E_{b i} F_{i}
\end{array}\right]\right\}
\end{aligned}
$$

Then, based on Lemma 2, an upper bound of $H_{11}$ obtained as:

$$
\begin{aligned}
& \sum_{k=1}^{r} \phi_{k}\left(P_{k}+R\right)+\bar{G}_{i i}^{T}\left(P_{k}+\mu R\right)+\left(P_{k}+\mu R\right) \bar{G}_{i i}+\lambda^{-1}\left(P_{k}+\mu R\right)\left[\begin{array}{ll}
D_{a i} & D_{b i}
\end{array}\right]\left[\begin{array}{l}
D_{a i}^{T} \\
D_{b i}^{T}
\end{array}\right] \\
& +\lambda\left[\begin{array}{ll}
E_{a i}^{T} & -\left(E_{b i} F_{i}\right)^{T}
\end{array}\right]\left[\begin{array}{l}
E_{a i} \\
-E_{b i} F_{i}
\end{array}\right]\left(P_{k}+\mu R\right) \prec 0
\end{aligned}
$$

by Schur complement, we obtain,

$$
\left[\begin{array}{ccc}
\Phi_{1} & \left(P_{k}+\mu R\right) D_{a i} & \left(P_{k}+\mu R\right) D_{b i} \\
* & -\lambda I & 0 \\
* & * & -\lambda I
\end{array}\right] \prec 0
$$

with

$$
\begin{gathered}
\Phi_{1}=P_{\phi}+\bar{G}_{i i}^{T}\left(P_{k}+\mu R\right)+\left(P_{k}+\mu R\right) \bar{G}_{i i}+\lambda\left(P_{k}+\mu R\right)\left[E_{a i}^{T} E_{a i}+\left(E_{b i} F_{i}\right)^{T} E_{b i} F_{i}\right] \\
\left\{\left(\frac{\bar{G}_{i j}+\bar{G}_{j i}}{2}\right)^{T}\left(P_{k}+\mu R\right)+\left(P_{k}+\mu R\right)\left(\frac{\bar{G}_{i j}+\bar{G}_{j i}}{2}\right)\right\}+\left\{\left(\left[\begin{array}{ll}
D_{a i}+D_{a j} & D_{b i}+D_{b j}
\end{array}\right]\left[\begin{array}{cc}
\Delta_{a i}+\Delta_{a j} & 0 \\
0 & \Delta_{b i}+\Delta_{b j}
\end{array}\right]\right.\right. \\
\left.\left.\times\left[\begin{array}{l}
E_{a i}+E_{a j} \\
-E_{b i} F_{j}-E_{b j} F_{i}
\end{array}\right]\right)^{T}\left(P_{k}+\mu R\right)+\left(P_{k}+\mu R\right) \times\left(\left[\begin{array}{ll}
D_{a i}+D_{a j} & D_{b i}+D_{b j}
\end{array}\right]\left[\begin{array}{cc}
\Delta_{a i}+\Delta_{a j} & 0 \\
0 & \Delta_{b i}+\Delta_{b j}
\end{array}\right] \times\left[\begin{array}{l}
E_{a i}+E_{a j} \\
-E_{b i} F_{j}-E_{b j} F_{i}
\end{array}\right]\right)\right\} \\
\prec 0
\end{gathered}
$$


Then, based on Lemma 2, an upper bound of $\Upsilon_{1}(x, z)$ obtained as:

$$
\begin{aligned}
& \left(\frac{\bar{G}_{i j}+\bar{G}_{j i}}{2}\right)^{T}\left(P_{k}+\mu R\right)+\left(P_{k}+\mu R\right)\left(\frac{\bar{G}_{i j}+\bar{G}_{j i}}{2}\right)+\lambda^{-1}\left(P_{k}+\mu R\right)\left[\begin{array}{ll}
D_{a i}+D_{a j} & D_{b i}+D_{b j}
\end{array}\right]\left[\begin{array}{c}
D_{a i}^{T}+D^{T}{ }_{a j} \\
D_{b i}^{T}+D^{T}{ }_{b j}
\end{array}\right] \\
& +\lambda\left[\left(E_{a i}+E_{a j}\right)^{T}\left(-E_{b i} F_{j}-E_{b j} F_{i}\right)^{T}\right] \times\left[\begin{array}{l}
E_{a i}+E_{a j} \\
-E_{b i} F_{j}-E_{b j} F_{i}
\end{array}\right]\left(P_{k}+\mu R\right) \prec 0
\end{aligned}
$$

by Schur complement, we obtain,

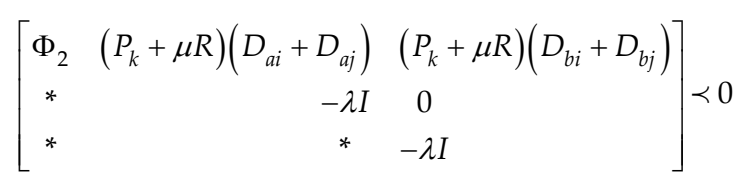

with

$$
\begin{aligned}
\Phi_{2} & =\left(\frac{\bar{G}_{i j}+\bar{G}_{j i}}{2}\right)^{T}\left(P_{k}+\mu R\right)+\left(P_{k}+\mu R\right)\left(\frac{\bar{G}_{i j}+\bar{G}_{j i}}{2}\right)+\lambda\left(P_{k}+\mu R\right)\left[\left(E_{a i}+E_{a j}\right)^{T}\left(E_{a i}+E_{a j}\right)\right. \\
& \left.+\left(E_{b i} F_{j}+E_{b j} F_{i}\right)^{T}\left(E_{b i} F_{j}+E_{b j} F_{i}\right)^{T}\right]
\end{aligned}
$$

If (49) and (50) holds, the time derivative of the fuzzy Lyapunov function is negative. Consequently, we have $\dot{V}(x(t)) \prec 0$ and the closed loop fuzzy system (5) is stable. This is complete the proof.

\section{Numerical examples}

Consider the following T-S fuzzy system:

$$
\dot{x}(t)=\sum_{i=1}^{r} h_{i}(z(t)) A_{i} x(t)
$$

with: $r=2$

the premise functions are given by:

$$
h_{1}\left(x_{1}(t)\right)=\frac{1+\sin x_{1}(t)}{2} ; h_{2}\left(x_{1}(t)\right)=\frac{1-\sin x_{1}(t)}{2} ; \quad A_{1}=\left[\begin{array}{ll}
-5 & -4 \\
-1 & -2
\end{array}\right] ; \quad A_{2}=\left[\begin{array}{ll}
-2 & -4 \\
20 & -2
\end{array}\right]
$$

It is assumed that $\left|x_{1}(t)\right| \leq \frac{\pi}{2}$. For $\xi_{11}=0, \xi_{12}=0.5, \quad \xi_{21}=-0.5$, and $\xi_{22}=0$, we obtain

$$
P_{1}=\left[\begin{array}{ll}
37.7864 & 26.8058 \\
26.8058 & 36.2722
\end{array}\right] ; \quad P_{2}=\left[\begin{array}{ll}
98.5559 & 28.7577 \\
28.7577 & 22.9286
\end{array}\right] ; \quad R=\left[\begin{array}{ll}
-1.2760 & -2.2632 \\
-2.2632 & -0.6389
\end{array}\right]
$$




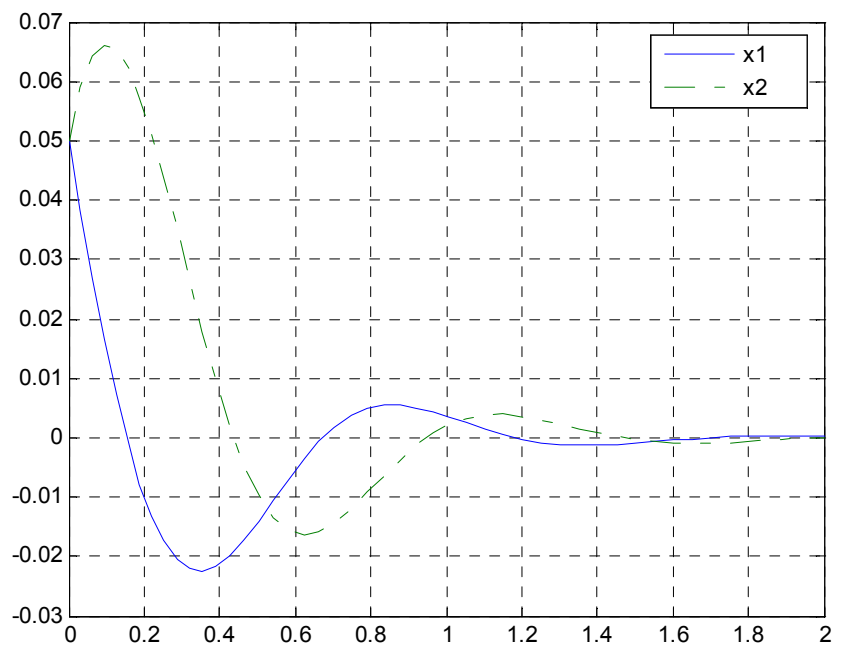

Figure 1. State variables

Figure 3 shows the evolution of the state variables. As can be seen, the conservatism reduction leads to very interesting results regarding fast convergence of this Takagi-Sugeno fuzzy system.

In order to show the improvements of proposed approaches over some existing results, in this section, we present a numerical example, which concern the feasibility of a time delay T$S$ fuzzy system. Indeed, we compare our fuzzy Lyapunov-Razumikhin approach (Theorem 3.1) with the Lemma 2.2 in [9].

Example 2. Consider the following T-S fuzzy system with $\mathrm{u}=0$ :

$$
\dot{x}(t)=\sum_{i=1}^{2} h_{i}(z(t))\left\{A_{i} x(t)+D_{i} x\left(t-\tau_{i}(t)\right)\right\}
$$

with:

$$
A_{1}=\left[\begin{array}{cc}
-2.1 & 0.1 \\
-0.2 & -0.9
\end{array}\right], A_{2}=\left[\begin{array}{cc}
-1.9 & 0 \\
-0.2 & -1.1
\end{array}\right], D_{1}=\left[\begin{array}{cc}
-1.1 & 0.1 \\
-0.8 & -0.9
\end{array}\right], D_{2}=\left[\begin{array}{cc}
-0.9 & 0 \\
-1.1 & -1.2
\end{array}\right],
$$

with the following membership functions :

$$
h_{1}=\sin ^{2}\left(x_{1}+0.5\right) ; \quad h_{2}=\cos ^{2}\left(x_{1}+0.5\right) \text {. }
$$

Assume that $\tau_{i}(t)=0.5\left|\sin \left(x_{1}(t)+x_{2}(t)+1\right)\right| \quad$ where $\quad x(t)=\left[x_{1}(t), x_{2}(t)\right]^{T} . \quad$ Then, $\tau_{i}(t) \leq \tau=0.5$. Table 1 . shows that our approach is less conservative than Lemma 2.2. given in [9]. 


\begin{tabular}{|c|c|}
\hline Methods & $\tau_{\max }$ \\
\hline Lemma 2.1 & 0.6308 \\
\hline Theorem 3.1 & $+\infty$ \\
\hline
\end{tabular}

Table 1. Comparison results of maximum $\tau$ for Example 1

The LMIs in (34)-(39) are feasible by choosing $X_{a i}=X_{a}, X_{d i}=X_{d}, Y_{i}=Y, Z_{a i j}=Z_{a}, Z_{d i j}=Z_{d}$, and $S_{i}=S, i, j=1,2$, and for $\tau=0.5$ a feasible solution is given by

$$
\begin{gathered}
P_{1}=\left[\begin{array}{cc}
1.5121 & -0.1801 \\
-0.1801 & 1.1057
\end{array}\right], P_{2}=\left[\begin{array}{cc}
1.451 & -0.178 \\
-0.178 & 0.883
\end{array}\right], S=\left[\begin{array}{cc}
1.021 & -0.064 \\
-0.064 & 0.664
\end{array}\right], \\
Y=\left[\begin{array}{cc}
-0.611 & 0.169 \\
-0.243 & -0.421
\end{array}\right], X_{a}=\left[\begin{array}{ll}
2.523 & 0.707 \\
0.707 & 2.155
\end{array}\right], X_{d}=\left[\begin{array}{cc}
1.448 & 0.094 \\
0.094 & 2.353
\end{array}\right], Z_{a}=\left[\begin{array}{cc}
0.201 & -0.087 \\
-0.087 & 0.369
\end{array}\right], \\
Z_{d}=\left[\begin{array}{cc}
0.849 & -0.227 \\
-0.227 & 0.246
\end{array}\right],
\end{gathered}
$$

\section{Conclusion}

This chapter provided new conditions for the stabilization with a PDC controller of TakagiSugeno fuzzy systems with time delay in terms of a combination of the Razumikhin theorem and the use of non-quadratic Lyapunov function as Fuzzy Lyapunov function. In addition, the time derivative of membership function is considered by the PDC fuzzy controller in order to facilitate the stability analysis. An approach to design an observer is derived in order to estimate variable states. In addition, a new condition of the stabilization of uncertain system is given in this chapter.

The stabilization condition proposed in this note is less conservative than some of those in the literature, which has been illustrated via examples.

\section{Author details}

Yassine Manai and Mohamed Benrejeb

National Engineering School of Tunis, LR-Automatique, Tunis, Tunisia

\section{References}

[1] T. Takagi, and M. Sugeno, "Fuzzy identification of systems and its application to modeling and control," IEEE Trans. On System, Man and Cybernetics, vol 15 (1), pp. 116132, 1985.

[2] M.A.L. Thathachar, P. Viswanah, "On the Stability of Fuzzy Systems", IEEE Transactions on Fuzzy Systems, Vol. 5, №1, pp. 145 - 151, February 1997. 
[3] L. K. Wong, F.H.F. Leung, P.K.S. Tam, "Stability Design of TS Model Based Fuzzy Systems", Proceedings of the Sixth IEEE International Conference on Fuzzy Systems, Vol. 1, pp. 83-86, 1997.

[4] C.H. Fang, Y.S. Liu, S.W. Kau, L. Hong, and C.H. Lee, “A New LMI-Based Approach to Relaxed Quadratic Stabilization of T-S Fuzzy Control Systems," IEEE Transactions on Fuzzy Systems, Vol. 14, N 3, pp.386-397, June 2006.

[5] H. K. Lam, F.H. Leung, "Stability Analysis of Fuzzy Model based Control Systems", Hong Kong, Springer 2011.

[6] I. Abdelmalek, N. Golea, and M.L. Hadjili, "A New Fuzzy Lyapunov Approach to NonQuadratic Stabilization of Takagi-Sugeno Fuzzy Models," Int. J. Appl. Math. Comput. Sci., Vol. 17, No. 1, 39-51, 2007.

[7] L.A. Mozelli, R.M. Palhares, F.O. Souza, and E.M. Mendes, "Reducing conservativeness in recent stability conditions of TS fuzzy systems," Automatica, Vol. 45, pp. 1580-1583, 2009.

[8] Y.Y. Cao and P.M. Frank, "Stability analysis and synthesis of nonlinear time-delay systems via Takagi-Sugeno fuzzy models", Fuzzy Sets and systems, Vol. 124 N², pp. 213-229, 2001.

[9] C. Lin, Q.G. Wang, T.H. Lee, "Delay-dependent LMI conditions for stability and stabilization of T-S fuzzy systems with bounded time-delay", Fuzzy Sets and Systems,

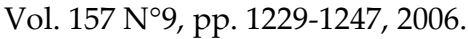

[10] C. Lin, Q.G. Wang, and T. H. Lee, “Fuzzy Weighting-dependent approach to $H_{\infty}$ filter design for Time-delay fuzzy systems", IEEE Transactions on Signal Processing, Vol. 55 $\mathrm{N}^{\circ}$ 6, 2007.

[11] C. Lin, Q.G. Wang, and T. H. Lee, LMI Approach to Analysis and Control of TakagiSugeno Fuzzy Systems with Time Delay, Springer-Verlag, Berlin 2007.

[12] Y.Y. Cao, and P.M. Frank. "Analysis and synthesis of nonlinear time-delay systems via fuzzy control approach". IEEE Transactions on Fuzzy Systems, 8(2), 200-211, 2000.

[13] I Amri, D Soudani, M Benrejeb, "Exponential Stability and Stabilization of Linear Systems with Time Varying Delays",Conf. SSD'09, Djerba, 2009.

[14] K. Tanaka, T. Hori, and H.O. Wang, "A multiple Lyapunov function approach to stabilization of fuzzy control systems," IEEE Transactions on Fuzzy Systems, Vol. 11 $\mathrm{N}^{\circ} 4$, pp. 582-589, 2003.

[15] C.H. Fang, Y.S. Liu, S.W. Kau, L. Hong, and C.H. Lee, “A New LMI-Based Approach to Relaxed Quadratic Stabilization of T-S Fuzzy Control Systems," IEEE Transactions on Fuzzy Systems, Vol. 14, N³, pp.386-397, June 2006.

[16] H.O. Wang, K. Tanaka, M. F. Griffin, "An Approach to Fuzzy Control of Nonlinear Systems: Stability and Design Issues", IEEE Transactions On Fuzzy Systems, Vol. 4, $\mathrm{N}^{\circ} 1$, February 1996.

[17] Y. Manai, M. Benrejeb, “Stability for Continuous Takagi-Sugeno Fuzzy System based on Fuzzy Lyapunov Function', Conf. CCCA'11, Hammamet, 2011.

[18] M. Yassine, B. Mohamed, "New Condition of Stabilisation for Continuous TakagiSugeno Fuzzy System based on Fuzzy Lyapunov Function", International Journal of Control and Automation, Vol. 4 No. 3, September, 2011. 